\title{
Cross-sectional study of patient-reported fatigue, physical activity and cardiovascular status in men after robotic-assisted radical prostatectomy
}

\author{
Ruth E. Ashton ${ }^{1}$ (D) Garry A. Tew ${ }^{1} \cdot$ Wendy A. Robson ${ }^{2} \cdot$ John M. Saxton $^{1} \cdot$ Jonathan J. Aning $^{3}$
}

Received: 24 October 2018 / Accepted: 31 March 2019/Published online: 10 April 2019

(C) The Author(s) 2019

\begin{abstract}
Purpose Patient-reported fatigue after robotic-assisted radical prostatectomy (RARP) has not been characterised to date. Fatigue after other prostate cancer (PCa) treatments is known to impact on patient-reported quality of life. The aim of this study was to characterise fatigue, physical activity levels and cardiovascular status post-RARP.

Methods Between October 2016 and March 2017, men post-RARP or on androgen deprivation therapy (ADT) were invited into the study. Participants were asked to complete the Brief Fatigue Inventory (BFI) and Stage of Change and Scottish Physical Activity Questionnaires (SPAQ) over a 2-week period. Outcome measures were patient-reported fatigue, physical activity levels and the 10-year risk of cardiovascular disease (Q-Risk). Data were analysed in SPSS.

Results 96/117 (82\%) men approached consented to participate; of these, 62/96 (65\%) returned complete questionnaire data (RARP $n=42$, ADT $n=20$ ). All men reported fatigue with 9/42 (21\%) post-RARP reporting clinically significant fatigue. Physical activity did not correlate with fatigue. On average, both groups were overweight (BMI $27.0 \pm 3.9 \mathrm{~kg} / \mathrm{m}^{2}$ and $27.8 \pm$ $12.3 \mathrm{~kg} / \mathrm{m}^{2}$ for RARP and ADT, respectively) and the post-RARP group had an $18.1 \% \pm 7.4 \%$ Q-Risk2 score.

Conclusions A proportion of men is at increased risk of cardiovascular disease within 10 years post-RARP and have substantial levels of fatigue; therefore, clinicians should consider including these factors when counselling patients about RARP. Additionally, men post-RARP did not meet the recommended guidelines for resistance-based exercise. Future research is needed to establish whether interventions including resistance-based exercise can improve health and fatigue levels in this population.
\end{abstract}

Keywords Prostate cancer $\cdot$ Prostatectomy $\cdot$ Fatigue $\cdot$ Physical activity

\section{Introduction}

Radical prostatectomy is an accepted curative treatment option for men with clinically localised significant prostate cancer ( $\mathrm{PCa})$ with greater than 10 years of life expectancy and the ability to perform activities of daily living [1]. Roboticassisted radical prostatectomy (RARP) is now the most

Ruth E. Ashton

rutheashton@gmail.com

1 Department of Sport, Exercise and Rehabilitation, University of Northumbria at Newcastle, Newcastle upon Tyne, UK

2 Department of Urology, Freeman Hospital, Newcastle upon Tyne, UK

3 Bristol Urological Institute, North Bristol NHS Trust, Southmead Hospital, Bristol, UK prevalent modality for surgical removal of the prostate for $\mathrm{PCa}$ in the UK [2].

RARP has been demonstrated to be associated with lower blood loss and decreased hospital stay when compared to open radical prostatectomy [3]. Although commonly assumed that men undergoing RARP are fit and return to their pre-operative physical activity levels after surgery, there is little published data to substantiate this assumption. The prevalence of fatigue and post-operative physical fitness in men who have undergone RARP is largely unknown with few studies performed to date have explored cancer-related fatigue post-RARP. What data there is suggests that fatigue is present in PCa patients but is affected by treatment modality and the time period over which fatigue is assessed; however, it has been previously reported that approximately $14 \%$ of patients who have undergone radical prostatectomy experience fatigue $[4,5]$. Fatigue in cancer patients and survivors has been associated with reduced physical activity levels [6], potentially adversely 
affecting cardiovascular risk profile and recovery to full functional fitness after RARP.

To our knowledge, no study has explored the association between self-reported physical activity, fatigue and comorbidities in men who have undergone RARP. The aim of this pilot study was to characterise fatigue, physical activity levels and cardiovascular status, over a 2 -week period, in men after RARP and establish whether this is a substantial problem, which future intervention studies should address.

\section{Methods}

\section{Design}

A cross-sectional questionnaire study was administered to men who had undergone RARP and men treated with ADT for PCa. Men on continuous medical ADT, a treatment strongly associated with a number of side effects that impact quality of life including significant fatigue [7], were purposely used as a comparative population with which to relate the morbidity of RARP. The study was approved by the South East Scotland NHS Research Ethics Committee. Data from the ADT cohort of men is presented as a comparative control population.

This study was conducted at Newcastle upon Tyne Hospitals NHS Foundation Trust which is a tertiary referral centre serving a population of 1.2 million people. RARP was performed by three experienced surgeons at the institution over the study period.

\section{Participants}

Men were eligible to participate in the study if they: (1) had histologically confirmed PCa, (2) were at least 8 weeks after their treatment for PCa with either RARP or after initiation of ADT, and (3) were able to provide consent and satisfactorily complete written questionnaires. All eligible patients attending outpatient's clinics were approached. Men receiving any other treatment for $\mathrm{PCa}$ were excluded from the study.

\section{Study outcome data}

Consenting men were asked to provide demographic information including current health status, average weekly alcohol intake and smoking status, and stature and body mass were measured. They were then invited to complete a questionnaire booklet containing validated questionnaires prospectively over a 2-week period (see further details below) and return the booklet in a prepaid stamp addressed envelope. Questionnaire score calculations were performed in accordance with published questionnaire protocols. Likewise, missing data were treated in accordance with the questionnaire protocols. The questionnaires included are detailed below.

\section{Comorbidity and cardiovascular status}

Charlson Comorbidity Index was calculated using information provided on stature, body mass and medical history [8, 9]. The risk of suffering a heart attack or stroke within the next 10 years was calculated using the validated objective measure: Q-Risk2 [10]. Q-Risk2 score is calculated from patient medical record data including family history, age, gender, ethnicity, socio-economic status, and selected physiological measurements, and can be categorised as $<10 \%$ (low), 10\%-20\% (medium) or $>20 \%$ (high) [11]. Q-Risk2 was specifically used in this study, because in addition to being the NICE recommended formal risk assessment tool for CVD, it is also an accepted aid to clinical decision-making regarding how intensively to intervene to improve health in patients with CVD [12].

\section{Scottish Physical Activity Questionnaire}

The Scottish Physical Activity Questionnaire (SPAQ) was completed at the end of both weeks as a recall questionnaire and has good reliability (Cronbach's alpha $=0.998)$ [13]. This questionnaire assesses moderate to vigorous physical activity (MVPA) over the previous 7 days. The questionnaire includes sections for both leisure time and occupational physical activity with each section containing questions on general activity such as walking, stair climbing and manual labour [13]. The average weekly total MVPA was calculated in addition to the mean total for each individual exercise component.

\section{Brief Fatigue Inventory}

The BFI was completed at the end of each day for all 14 days of the data collection period to rapidly assess fatigue in cancer patients and is correlated with other validated fatigue questionnaires [14-16] and has good reliability (Cronbach's alpha $=0.95)$ [16]. The BFI consists of three questions assessing fatigue severity and six questions assessing the interference of fatigue with the patient's mood and social/physical functioning with all answers being on a $0-10$ scale. A global fatigue score was obtained for weeks 1 and 2 by averaging all the items on the BFI and as an average of the whole 2-week period [16]. Clinically significant fatigue is defined as a global fatigue score $>3[5,17]$.

\section{Stage of Change Questionnaire}

The Stage of Change Questionnaire was administered once at the start of the 2-week study period to assess patient's attitudes towards exercise behaviour change and has acceptable reliability (Cronbach's alpha $=0.63)[18]$. Participants answered 'yes' or 'no' to four statements to assess each individual's stage of behaviour change [6]. The stages are categorised as 
follows: stage 1-pre-contemplation, stage 2-contemplation, stage 3-preparation, stage 4-action and, and stage 5-maintenance.

\section{Statistical analysis}

All returned surveys were included in the analysis, even if some sections were incomplete. Consequently, the number of total responses for each survey item varied because of missing data. Analyses were conducted using IBM SPSS Statistics Version 22 (IBM United Kingdom Limited, Hampshire, UK).

Normality was assessed using the Shapiro-Wilk and, if data was not normally distributed, transformations were conducted using common logarithms or square root. To assess the associations of the outcomes with self-reported total PA levels (SPAQ), Pearson correlations and Spearman's rank were employed. Independent samples $t$ tests were used to examine differences between the two treatment groups with $p<0.05$ chosen as the accepted level of significance.

\section{Results}

\section{Participants}

In total, 148 men were approached to take part in the study and 96 men consented to participate in the study; of these, 62/96 $(65 \%)$ patients returned postal questionnaires. Table 1

Table 1 Patient demographics

\begin{tabular}{lll}
\hline & RARP $(n=42)$ & ADT $(n=20)$ \\
\hline Age (years) & $63.8 \pm 6.4$ & $67.3 \pm 9.0$ \\
Body mass $(\mathrm{kg})$ & $86.7 \pm 13.4$ & $86.4 \pm 12.3$ \\
Stature $(\mathrm{cm})$ & $180 \pm 0.07$ & $176 \pm 0.07$ \\
Body mass index $\left(\mathrm{kg} / \mathrm{m}^{2}\right)$ & $27.0 \pm 3.9$ & $27.8 \pm 12.3$ \\
Drink alcohol $n(\%)$ & $38(90.5)$ & $18(80.0)$ \\
Months since treatment mean (range) & $11.7(2-115)$ & $22.1(2-120)$ \\
Pre-RARP PSA & $10.05 \pm 6.3$ & \\
Pathological Gleason Score $(n)$ & & \\
GS 6 & 2 & \\
GS 3+4 & 25 & \\
GS 4+ 3 & 7 & \\
GS $\geq 8$ & 8 & \\
Pathological tumour stage $(n)$ & & \\
PT2 & 24 & \\
PT3a & 13 & \\
PT3b & 5 &
\end{tabular}

Data are presented as mean \pm standard deviation unless stated otherwise $R A R P$ robot-assisted radical prostatectomy, $A D T$ androgen deprivation therapy, $P S A$ prostate specific androgen illustrates the demographic of the cohort. The patients approached were on average 11.7 months after RARP and 22.1 months after the initiation of ADT. The RARP cohort comprised $42 / 62$ responses; of these, $57 \%$ and $14 \%$ were classified as overweight and obese, respectively.

\section{Cardiovascular status}

Charlson Comorbidity Indexcalculations indicated that there was no significant difference in estimated 10-year survival after $\operatorname{RARP}(87.3 \% \pm 12.2 \%)$ or $\operatorname{ADT}(80.5 \% \pm 18.7 \%)$, $t(27.2)=1.5, p=0.2$. Q-Risk2 scores indicated that there was no significant difference in 10-year risk of suffering a heart attack or stroke between men post-RARP $(18.1 \% \pm$ $7.4 \%)$ and after initiation of $\operatorname{ADT}(22.4 \% \pm 10.8 \%)$, $t(28.4)=-1.6, p=0.12$.

\section{Physical activity}

The levels of reported PA did not differ over the 2-week period between the two treatment groups (RARP total average mins $=658.1 \pm 337.6$ versus ADT total average mins $=$ $631.9 \pm 318.5, t(59)=0.3, p=0.8)$. Age, body mass, BMI and BFI scores were not associated with the total amount of PA performed in either treatment group (Table 2). Approximately $50 \%$ of all PA reported in both groups involved walking (e.g. walking to the shops/work, stair walking). Activities included in the 'other' category included yoga (1/42 post-RARP, 1/20 ADT), bowls (1/42 post-RARP) and rambling (2/42 post-RARP, 1/20 ADT). A breakdown of the amount of physical activity undertaken is illustrated in Table 3.

\section{Fatigue}

All patients were experiencing fatigue over the 2-week study period; the majority of fatigue reported was mild-moderate in severity and of borderline clinical significance. The mean

Table 2 Correlation matrix between physical activity and demographic factors, comorbidities, stage of change and fatigue

\begin{tabular}{lll}
\hline & \multicolumn{2}{l}{ Physical activity $^{\mathrm{a}}$} \\
\cline { 2 - 3 } & RARP & ADT \\
\hline Age & -0.1 & -0.14 \\
Body mass & -0.02 & -0.31 \\
Body mass index & 0.1 & -0.09 \\
Stage of change & $0.36^{\mathrm{b}}$ & 0.15 \\
Brief Fatigue Inventory & -0.09 & 0.09 \\
\hline
\end{tabular}

${ }^{\text {aTotal }}$ physical activity in minutes averaged over the 2-week study period

${ }^{\mathrm{b}}$ Correlation is significant at the 0.05 level 
Table 3 Self-reported MVPA over the 2-week period

\begin{tabular}{lll}
\hline & RARP & ADT \\
\hline Total (mins) & $658.1 \pm 337.6$ & $631.9 \pm 318.5$ \\
Walking (mins) & $341.4 \pm 245.5$ & $319.5 \pm 251.4$ \\
Manual labour (mins) & $125.4 \pm 168.3$ & $92.3 \pm 158.2$ \\
Active housework (mins) & $57.6 \pm 79.6$ & $82.4 \pm 89.4$ \\
Dancing (mins) & $3.2 \pm 11.3$ & $0.63 \pm 2.8$ \\
Sport/Leisure activities (mins) & $92.3 \pm 178.5$ & $126.4 \pm 209.4$ \\
Other activities (mins) & $40.4 \pm 100.2$ & $6.3 \pm 22.3$ \\
\hline
\end{tabular}

Data are presented as mean \pm standard deviation

Two RARP participants did not provide physical activity data for week 1 or week 2

severity of fatigue was significantly less over the 2-week study period in the RARP $(1.6 \pm 1.7)$ than in the ADT group $(2.6 \pm$ 1.8), $t(60)=-2.628, p=0.011$ (Fig. 1). However, 9/42 (21.4\%) patients' post-RARP and 6/20 (30\%) ADT patients reported clinically significant fatigue. There was no association between fatigue and the amount of self-reported PA (Table 2).

\section{Stage of change}

The majority of patients in both treatment groups reported being in the maintenance stage of change (Table 4). The maintenance phase is where individuals have made specific modifications to their exercise behaviour; however, it requires a conscious effort to in order to maintain it. The stage of change outcomes positively correlated with the amount of PA undertaken in the RARP treatment group; this is shown in Table 2.

\section{Discussion}

This is the first study to our knowledge to quantitatively explore cardiovascular risk, fatigue and physical activity, in men who have undergone RARP as a PCa treatment. Our study

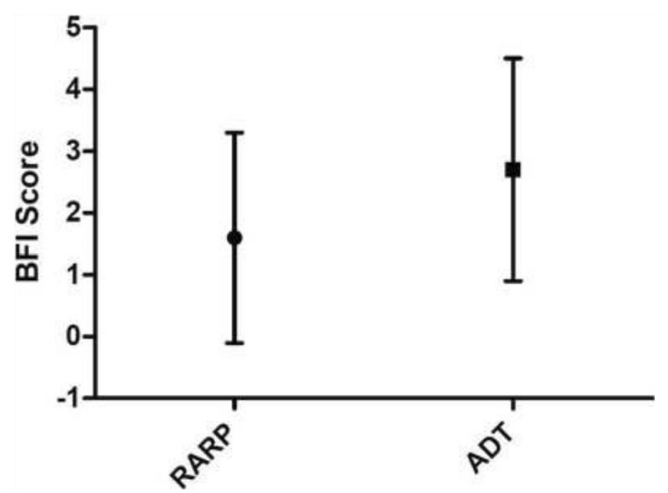

Fig. 1 Brief Fatigue Inventory scores presented as a global score (range of scores 1-6) for RARP and ADT over the 2-week study period
Table 4 Stage of change scores for both treatment groups

\begin{tabular}{lll}
\hline & $\operatorname{RARP}(n=42)$ & $\mathrm{ADT}(n=20)$ \\
\hline \multicolumn{2}{l}{ Stage of change (number of patients) } & \\
Stage 1-pre-contemplation & 0 & 0 \\
Stage 2-contemplation & 1 & 3 \\
Stage 3-preparation & 0 & 1 \\
Stage 4- action & 5 & 3 \\
Stage 5-maintenance & 36 & 13 \\
\hline
\end{tabular}

found that a substantial proportion of men post-RARP is at increased risk of suffering a cardiovascular related event within 10-15 years of their surgery and may suffer from clinically significant fatigue. Importantly, this study demonstrated that these findings were present in men appearing to meet the UK public health guidance of undertaking at least $150 \mathrm{~min}$ a week of moderate to vigorous aerobic physical activity. There was no significant difference between the cardiovascular risks found in men post-RARP and those on ADT in this study.

The present study identified that the Q-Risk2 score of the RARP cohort indicated that they were at a $18 \%$ greater risk of suffering a heart attack or stroke within the next 10 years. Whilst cardiovascular risk status has been studied extensively in patients receiving ADT [19-22], there are no studies, as far as the authors are aware to date, which have characterised the cardiovascular risk status of RARP patients. The largest most contemporary study by Wilt et al. [23] gives a signal as to mortality risk from causes other than $\mathrm{PCa}$ in a radical prostatectomy population. During a median follow-up of period of 10 years, 171/281 of the radical prostatectomy group died, and of these deaths, 74\% (127/171) were not due to PCa [23]. Whilst it cannot be inferred that cardiovascular disease was the cause of all of these deaths due to other factors such as old age, this analysis combined with our study findings indicate potentially more can be done to improve the health of patients undergoing RARP. The Q-Risk2 calculator aids clinical decision-making about how intensively to recommend lifestyle interventions and lipid-lowering medications to patients with significant cardiovascular risks during patient-centred consultations [10]. The results of our study showed that our RARP cohort was at a $3 \%$ elevated risk of cardiovascular disease when compared to moderately active males of a similar age [24]. This finding, where it to be replicated in future studies, supports the view that men after RARP should at the very least be informed of their risk which could increase their compliance when offered lifestyle interventions to improve their cardiovascular health $[25,26]$.

Cancer-related fatigue has previously been reported as a side effect of PCa treatment in up to $80 \%$ of men [27-30]. Few studies have investigated levels of fatigue in men who have exclusively undergone RARP for PCa; however, much work has been conducted in men receiving ADT and 
radiotherapy. Storey et al. [5] performed a cross-sectional questionnaire study of recurrence free survivors who had undergone open radical prostatectomy utilising the BFI. Clinically relevant fatigue was identified in $22 \%$ (29/133) of men undergoing radical prostatectomy, whereas in their control non-cancer population, the incidence of clinically relevant fatigue was $16 \%(10 / 63)$ at a median follow-up of 56 months after treatment. Within their radical prostatectomy cohort median age 72, coexisting depression had the strongest independent association with fatigue. Storey et al. [5] did not examine PA levels within their cohort. Cancer-related fatigue has been acknowledged to be debilitating and to significantly impact on quality of life [31]. We have shown that after RARP in a contemporary younger population, similar to Storey et al., clinically relevant fatigue is reported by $20 \%$ of men at a mean follow-up of 11.7 months. This finding might be considered unexpected but highlights that post-treatment fatigue should be discussed with patients when they are counselled for RARP. All patients included in our study underwent holistic needs assessment after treatment and received targeted support if required as part of routine care from a survivorship nurse specialist $[32,33]$. We have previously demonstrated that men in our institution who undergo RARP experience an unchanged overall quality of life [32]; therefore, it is probable that the clinically relevant fatigue identified in this study is unlikely to have resulted from psychological factors.

As far as the authors are aware, this is the first examination of PA sub-classifications undertaken in a contemporary population of men who have undergone RARP. Our study found patient-reported levels of PA after RARP which met current UK public health guidelines within the RARP cohort despite a high proportion of our patients having a high body mass index. We demonstrated that PA levels did not correlate with fatigue levels suggesting that fatigue levels may not be a barrier to the amount of PA undertaken within this population. Although public health guideline levels of aerobic PA were met, we identified that post-RARP patients did not achieve the recommended weekly amount of resistance exercise [34], with none of the patients reporting completing any resistance exercise. This important finding highlights a potential area of unmet need in the post-RARP population. Resistance exercise has previously been examined in other PCa treatment groups, both epidemiologically and during interventional studies which found resistance exercise to be safe in the population, alongside mitigating fatigue and generating longer-term improvements in quality of life, strength, triglycerides and body fat when compared to aerobic exercise $[28,35,36]$. The potential benefits of resistance exercise in relation to cardiometabolic risk profile were highlighted in a recent meta-analysis [37]. Although loss of skeletal muscle mass has been widely reported in PCa patients undergoing ADT [38-40] and many studies have investigated the impact of resistance exercise training programmes $[35,39,41]$, much fewer studies have assessed changes in skeletal muscle characteristics after RARP. There is a need for future research to address this evidence gap, and extending the provision of structured exercise interventions (including resistance exercise) to this population may be warranted. Such interventions could have a positive impact on fatigue in men recovering from RARP, as demonstrated previously in fatigued $\mathrm{PCa}$ patients receiving ADT [29].

This study's findings add quantitative depth to recent qualitative work performed by Sutton et al. [42] and HackshawMcGeagh et al. [43] identifying patients' priorities. These studies showed that men undergoing RARP would value PA and dietary advice from their healthcare professional and would prefer to receive this at an early stage. In addition, they provided evidence that men undergoing RARP are willing to change their behaviour to improve their health, but they wish to be supported by their healthcare professional team to do so. Undergoing RARP is potentially a 'teachable moment,' and we have demonstrated that this population is at risk of both cardiovascular events and fatigue. Qualitative research shows men are receptive to health behaviour change [42-44], and such initiatives targeted at this population could have much potential to improve men's overall health.

Our study supports consideration of further targeted research into strategies aimed at improving the health of men who have undergone RARP. Feasibility to recruit patients and compliance with completing study questionnaires has been demonstrated, in addition to the ability to discriminate the health status and behaviours of the RARP population. The present study has limitations. Whilst the patient-reported questionnaire data showed that all men appeared to meet UK guidance physical activity levels, the authors did not expect this finding. Additionally, we identified that the SPAQ does not allow for the separation of exercise at varying intensities, and therefore, potential over reporting of the amount of MVPA may take place. For example, within the walking category, some low intensity physical activity may have been included despite the instructions stating otherwise. We believe that our findings justify the inclusion of activity trackers used in parallel with patient-reported activity questionnaires in future study protocols to strengthen validity of activity outcome results. Our study did not investigate participants for sleep disorders. Although non-restorative sleep and fatigue are different entities, we acknowledge symptoms described by patients with each of these conditions may be similar and this should be investigated in future studies with a measure of sleep quality used alongside fatigue questionnaires [45]. In this study, we did not use a group of healthy men without cancer who had not undergone surgery as a comparator group and accept that this might be considered as a limitation. Patient and Public Involvement was integral to the design of this study. Prostate cancer patients felt that it was assumed that, because they were undergoing RARP, they were fit and that there was a lack of 
recommendations and guidance regarding fatigue, and health and lifestyle improvements they could make after RARP that should be addressed. This study was pragmatically designed in response to specific feedback from the Newcastle upon Tyne Patient and Public Involvement Group who felt that the comparator should be what they considered the most morbid prostate cancer treatment continuous ADT on the basis that, if similar morbidities were demonstrated, this would reinforce the need for targeted interventions in men after RARP. We acknowledge however that cardiovascular risk and fatigue will be present in the general healthy population. Finally, although the numbers included in this study were small, it has identified the need for further study in this population of men and informed the sample size calculation required for further work in this area. In order to conduct a fully powered study using the reported fatigue effect size from this pilot study (Cohen's $d=0.57$ ), the minimum total sample size to achieve $80 \%$ power $(\alpha=0.05)$ was determined as $n=100$ patients ( 50 patients in each group) would be needed to detect differences in fatigue between the two groups [46].

\section{Conclusion}

Our study has shown that some men post-RARP are at increased risk of clinically significant consequences from cardiovascular disease within 10 years of their surgery and do suffer with clinically significant levels of fatigue. Clinicians should consider including these factors in the discussion when counselling patients about RARP. We have shown that men after RARP appear to meet the recommended guidelines for aerobic physical activity but do not meet them for resistancebased exercise. Future research is needed to establish whether exercise interventions can improve health and fatigue levels in this population.

Acknowledgements Stephen Brown, a patient representative, was an integral part in designing and guiding this study. Further acknowledgement should be given to the Newcastle upon Tyne Patient and Public Involvement Group for their support and valuable feedback.

Funding This study was funded by The Urology Foundation.

\section{Compliance with ethical standards}

Conflict of interest The authors declare that they have no conflict of interest.

Open Access This article is distributed under the terms of the Creative Commons Attribution 4.0 International License (http:// creativecommons.org/licenses/by/4.0/), which permits unrestricted use, distribution, and reproduction in any medium, provided you give appropriate credit to the original author(s) and the source, provide a link to the Creative Commons license, and indicate if changes were made.

\section{References}

1. National Institute for Health and Care Excellence (2014) Prostate cancer: diagnosis and managment. Clinical guideline [CG175]

2. Khadhouri S, Miller C, Fowler S, Hounsome L, McNeill A, Adshead J, McGrath JS (2018) The British Association of Urological Surgeons (BAUS) radical prostatectomy audit 2014/ 2015 - an update on current practice and outcomes by centre and surgeon case-volume. BJU Int 121(6):886-892. https://doi.org/10. 1111/bju.14156

3. Djavan B, Eckersberger E, Finkelstein J, Sadri H, Farr A, Apolikhin O, Lepor H (2010) Oncologic, functional, and cost analysis of open, laparoscopic, and robotic radical prostatectomy. Eur Urol Suppl 9(3):371-378. https://doi.org/10.1016/j.eursup.2010.02.009

4. Köhler N, Gansera L, Holze S, Friedrich M, Rebmann U, Stolzenburg J-U, Truss MC, Fahlenkamp D, Scholz H-J, Brähler E (2014) Cancer-related fatigue in patients before and after radical prostatectomy. Results of a prospective multi-centre study. Support Care Cancer 22(11):2883-2889. https://doi.org/10.1007/s00520014-2265-5

5. Storey DJ, McLaren DB, Atkinson MA, Butcher I, Liggatt S, O'Dea R, Smyth JF, Sharpe M (2012) Clinically relevant fatigue in recurrence-free prostate cancer survivors. Ann Oncol 23(1):6572. https://doi.org/10.1093/annonc/mdr034

6. Blaney JM, Lowe-Strong A, Rankin-Watt J, Campbell A, Gracey JH (2013) Cancer survivors' exercise barriers, facilitators and preferences in the context of fatigue, quality of life and physical activity participation: a questionnaire-survey. Psychooncology 22(1):186194. https://doi.org/10.1002/pon.2072

7. Higano CS (2003) Side effects of androgen deprivation therapy: monitoring and minimizing toxicity. Urology 61(2 Suppl 1):32-38

8. Charlson ME, Pompei P, Ales KL, MacKenzie CR (1987) A new method of classifying prognostic comorbidity in longitudinal studies: development and validation. J Chronic Dis 40(5):373-383

9. MDCalc (2018) Charlson Comorbidity Index (CCI). https://www. mdcalc.com/charlson-comorbidity-index-cci. Accessed 20-6-2018 2018

10. Hippisley-Cox J, Coupland C, Vinogradova Y, Robson J, Minhas R, Sheikh A, Brindle P (2008) Predicting cardiovascular risk in England and Wales: prospective derivation and validation of QRISK2. Bmj 336(7659):1475-1482. https://doi.org/10.1136/ bmj.39609.449676.25

11. Coghill N, Garside L, Montgomery AA, Feder G, Horwood J (2018) NHS health checks: a cross- sectional observational study on equity of uptake and outcomes. BMC Health Serv Res 18:238. https://doi.org/10.1186/s12913-018-3027-8

12. National Institute for Health and Care Excellence (2015) Cardiovascular Risk Assessment and Lipid Modification: Quality standard [QS100]. NICE. https://www.nice.org.uk/guidance/ qs100/resources

13. Lowther M, Mutrie N, Loughlan C, McFarlane C (1999) Development of a Scottish physical activity questionnaire: a tool for use in physical activity interventions. Br J Sports Med 33(4): 244-249

14. Ahlberg K, Ekman T, Gaston-Johansson F, Mock V (2003) Assessment and management of cancer-related fatigue in adults. Lancet 362(9384):640-650. https://doi.org/10.1016/s01406736(03)14186-4

15. Sternberg CN, Molina A, North S, Mainwaring P, Fizazi K, Hao Y, Rothman M, Gagnon DD, Kheoh T, Haqq CM, Cleeland C, de Bono JS, Scher HI (2013) Effect of abiraterone acetate on fatigue in patients with metastatic castration-resistant prostate cancer after docetaxel chemotherapy. Ann Oncol 24(4):1017-1025. https://doi. org/10.1093/annonc/mds585 
16. Mendoza TR, Wang XS, Cleeland CS, Morrissey M, Johnson BA, Wendt JK, Huber SL (1999) The rapid assessment of fatigue severity in cancer patients: use of the Brief Fatigue Inventory. Cancer 85(5):1186-1196

17. Shafqat A, Einhorn LH, Hanna N, Sledge GW, Hanna A, Juliar BE, Monahan P, Bhatia S (2005) Screening studies for fatigue and laboratory correlates in cancer patients undergoing treatment $\dagger$. Ann Oncol 16(9):1545-1550. https://doi.org/10.1093/annonc/mdi267

18. Donovan RJ, Jones S, Holman CDAJ, Corti B (1998) Assessing the reliability of a stage of change scale. Health Educ Res 13(2):285291. https://doi.org/10.1093/her/13.2.285

19. Gilbert SE, Tew GA, Bourke L, Winter EM, Rosario DJ (2013) Assessment of endothelial dysfunction by flow-mediated dilatation in men on long-term androgen deprivation therapy for prostate cancer. Exp Physiol 98(9):1401-1410. https://doi.org/10.1113/ expphysiol.2013.073353

20. Keating NL (2017) Type of androgen deprivation therapy and risk of cardiovascular disease. Eur Urol 72(6):929-930. https://doi.org/ 10.1016/j.eururo.2017.08.004

21. Voog JC, Paulus R, Shipley WU, Smith MR, McGowan DG, Jones CU, Bahary JP, Zeitzer KL, Souhami L, Leibenhaut MH, Rotman M, Husain SM, Gore E, Raben A, Chafe S, Sandler HM, Efstathiou JA (2016) Cardiovascular mortality following short-term androgen deprivation in clinically localized prostate cancer: an analysis of RTOG 94-08. Eur Urol 69(2):204-210. https://doi.org/10.1016/j. eururo.2015.08.027

22. Wallis CJ, Mahar AL, Satkunasivam R, Herschorn S, Kodama RT, Lee Y, Kulkarni GS, Narod SA, Nam RK (2016) Cardiovascular and skeletal-related events following localized prostate cancer treatment: role of surgery, radiotherapy, and androgen deprivation. Urology 97:145-152. https://doi.org/10.1016/j.urology.2016.08. 002

23. Wilt TJ, Brawer MK, Jones KM, Barry MJ, Aronson WJ, Fox S, Gingrich JR, Wei JT, Gilhooly P, Grob BM, Nsouli I, Iyer P, Cartagena R, Snider G, Roehrborn C, Sharifi R, Blank W, Pandya P, Andriole GL, Culkin D, Wheeler T (2012) Radical prostatectomy versus observation for localized prostate cancer. N Engl J Med 367(3):203-213. https://doi.org/10.1056/NEJMoa1113162

24. West J (2015) Does physical inactivity increase personalised cardiovascular risk in males and females aged 40-80 years? 20158 (1):21

25. Sheridan SL, Viera AJ, Krantz MJ, Ice CL, Steinman LE, Peters KE, Kopin LA, Lungelow D (2010) The effect of giving global coronary risk information to adults: a systematic review. Arch Intern Med 170(3):230-239. https://doi.org/10.1001/ archinternmed.2009.516

26. Casebeer L, Huber C, Bennett N, Shillman R, Abdolrasulnia M, Salinas GD, Zhang S (2009) Improving the physician-patient cardiovascular risk dialogue to improve statin adherence. BMC Fam Pract 10:48. https://doi.org/10.1186/1471-2296-10-48

27. Stone P, Richardson A, Ream E, Smith AG, Kerr DJ, Kearney N (2000) Cancer-related fatigue: inevitable, unimportant and untreatable? Results of a multi-centre patient survey. Cancer Fatigue Forum. Ann Oncol 11(8):971-975

28. Segal RJ, Reid RD, Courneya KS, Sigal RJ, Kenny GP, Prud'Homme DG, Malone SC, Wells GA, Scott CG, Slovinec D'Angelo ME (2009) Randomized controlled trial of resistance or aerobic exercise in men receiving radiation therapy for prostate cancer. J Clin Oncol 27(3):344-351. https://doi.org/10.1200/JCO. 2007.15.4963

29. Taaffe DR, Newton RU, Spry N, Joseph D, Chambers SK, Gardiner RA, Wall BA, Cormie P, Bolam KA, Galvao DA (2017) Effects of different exercise modalities on fatigue in prostate cancer patients undergoing androgen deprivation therapy: a year-long randomised controlled trial. Eur Urol 72(2):293-299. https://doi.org/10.1016/j. eururo.2017.02.019

30. Luo H-C, Lei Y, Cheng H-H, Fu Z-C, Liao S-G, Feng J, Yin Q, Chen Q-H, Lin G-S, Zhu J-F, Xu J-F, Wang D (2016) Long-term cancer-related fatigue outcomes in patients with locally advanced prostate cancer after intensity-modulated radiotherapy combined with hormonal therapy. Medicine 95(25):e3948. https://doi.org/10. 1097/MD.0000000000003948

31. Berger AM, Mooney K, Alvarez-Perez A, Breitbart WS, Carpenter KM, Cella D, Cleeland C, Dotan E, Eisenberger MA, Escalante CP, Jacobsen PB, Jankowski C, LeBlanc T, Ligibel JA, Loggers ET, Mandrell B, Murphy BA, Palesh O, Pirl WF, Plaxe SC, Riba MB, Rugo HS, Salvador C, Wagner LI, Wagner-Johnston ND, Zachariah FJ, Bergman MA, Smith C (2015) Cancer-related fatigue, version 2.2015. J Natl Compr Cancer Netw 13(8):1012-1039

32. Aning JJ, MacKenzie KR, Fabricius M, McColl E, Johnson MI, Tandogdu Z, Soomro NA, Harding C (2018) Detailed analysis of patient-reported lower urinary tract symptoms and effect on quality of life after robotic radical prostatectomy. Urol Oncol 36(8): 364.e315-364.e322. https://doi.org/10.1016/j.urolonc.2018.05.017

33. Ferguson J, Aning J (2015) Prostate cancer survivorship: a nurseled service model. Br J Nurs 24(Sup18):S14-S21. https://doi.org/ 10.12968/bjon.2015.24.Sup18.S14

34. Department of Health (2011) Factsheet 4: physical activity guidelines for adults (19-64 years) https://www.gov.uk/government/ publications/uk-physical-activity-guidelines

35. Segal RJ, Reid RD, Courneya KS, Malone SC, Parliament MB, Scott CG, Venner PM, Quinney HA, Jones LW, D'Angelo MES, Wells GA (2003) Resistance exercise in men receiving androgen deprivation therapy for prostate cancer. J Clin Oncol 21(9):16531659. https://doi.org/10.1200/jco.2003.09.534

36. Cormie P, Newton RU, Spry N, Joseph D, Taaffe DR, Galvao DA (2013) Safety and efficacy of resistance exercise in prostate cancer patients with bone metastases. Prostate Cancer Prostatic Dis 16(4): 328-335. https://doi.org/10.1038/pcan.2013.22

37. Ashton RE, Tew GA, Aning JJ, Gilbert SE, Lewis L, Saxton JM (2018) Effects of short-term, medium-term and long-term resistance exercise training on cardiometabolic health outcomes in adults: systematic review with meta-analysis. Br J Sports Med. https://doi.org/ 10.1136/bjsports-2017-098970

38. Owen PJ, Daly RM, Livingston PM, Mundell NL, Dalla Via J, Millar JL, Fraser SF (2017) Efficacy of a multi-component exercise programme and nutritional supplementation on musculoskeletal health in men treated with androgen deprivation therapy for prostate cancer (IMPACT): study protocol of a randomised controlled trial. Trials 18(1):451. https://doi.org/10.1186/s13063-017-2185-Z

39. Winters-Stone KM, Dieckmann N, Maddalozzo GF, Bennett JA, Ryan CW, Beer TM (2015) Resistance exercise reduces body fat and insulin during androgen-deprivation therapy for prostate cancer. Oncol Nurs Forum 42(4):348-356. https://doi.org/10.1188/15. onf.348-356

40. Galvao DA, Taaffe DR, Spry N, Joseph D, Turner D, Newton RU (2009) Reduced muscle strength and functional performance in men with prostate cancer undergoing androgen suppression: a comprehensive cross-sectional investigation. Prostate Cancer Prostatic Dis 12(2):198-203. https://doi.org/10.1038/pcan.2008.51

41. Hasenoehrl T, Keilani M, Sedghi Komanadj T, Mickel M, Margreiter M, Marhold M, Crevenna R (2015) The effects of resistance exercise on physical performance and health-related quality of life in prostate cancer patients: a systematic review. Support Care Cancer 23(8):2479-2497. https://doi.org/10.1007/s00520015-2782-x

42. Sutton E, Hackshaw-McGeagh LE, Aning J, Bahl A, Koupparis A, Persad R, Martin RM, Lane JA (2017) The provision of dietary and 
physical activity advice for men diagnosed with prostate cancer: a qualitative study of the experiences and views of health care professionals, patients and partners. Cancer Causes Control 28(4):319329. https://doi.org/10.1007/s10552-017-0861-7

43. Hackshaw-McGeagh LE, Sutton E, Persad R, Aning J, Bahl A, Koupparis A, Millett C, Martin RM, Lane JA (2017) Acceptability of dietary and physical activity lifestyle modification for men following radiotherapy or radical prostatectomy for localised prostate cancer: a qualitative investigation. BMC Urol 17:94. https://doi.org/10.1186/s12894-017-0284-5

44. Er V, Lane JA, Martin RM, Persad R, Chinegwundoh F, Njoku V, Sutton E (2017) Barriers and facilitators to healthy lifestyle and acceptability of a dietary and physical activity intervention among
African Caribbean prostate cancer survivors in the UK: a qualitative study. BMJ Open 7(10):e017217. https://doi.org/10.1136/bmjopen2017-017217

45. Roscoe JA, Kaufman ME, Matteson-Rusby SE, Palesh OG, Ryan JL, Kohli S, Perlis ML, Morrow GR (2007) Cancer-related fatigue and sleep disorders. Oncologist 12(Suppl 1):35-42. https://doi.org/ 10.1634/theoncologist.12-S1-35

46. Cohen J (1977) Statistical power analysis for the behavioral sciences, 1st edn. Academic Press, Cambridge

Publisher's note Springer Nature remains neutral with regard to jurisdictional claims in published maps and institutional affiliations. 